\section{Revista de CIENCIAS AMBIENTALES Tropical Journal of Environmental Sciences}

Revista de Ciencias Ambientales (Trop J Environ Sci)

e-ISSN: 2215-3896

(Enero-Junio, 2022) . Vol 56(1): 75-95

DOI: https://doi.org/10.15359/rca.56-1.4

Open Access: www.revistas.una.ac.cr/ambientales e-mail: revista.ambientales@una.ac.cr Rodríguez Cueto Y., Ramón Puebla A.

\title{
Evaluación de modelos para generar rutas de conectividad entre áreas naturales protegidas en Granma, Cuba
}

\author{
Evaluation of models to generate connectivity routes between protected natural \\ areas in Granma, Cuba
}

\author{
Yandy Rodríguez Cueto ${ }^{1}$, Adonis Ramón Puebla ${ }^{2}$
}

[Recibido: 3 de septiembre 2021, Aceptado: 21 de octubre 2021, Corregido: 10 de noviembre 2021, Publicado: 1 de enero 2022]

\section{Resumen}

[Introducción]: La mayoría de las investigaciones en generación de rutas de conectividad emplean mapas de costos al desplazamiento generados a partir de la reclasificación en rangos de las variables empleadas. [Objetivo]: En esta investigación se evalúa el efecto de la selección del método de generación del ráster de costos al desplazamiento sobre el trazado de las potenciales rutas de conectividad entre parches de bosque en las áreas naturales protegidas de la provincia Granma, Cuba. [Metodología]: Se generan dos ráster de costos al desplazamiento (uno con rangos y otro sin rangos) para los cuales se obtienen las potenciales rutas de conectividad y se analiza el efecto del método de cálculo del ráster de costo sobre el diseño de las rutas. [Resultados]: Se identifican un total de 3080 rutas. La densidad de rutas para el ráster con rangos no sobrepasa las 1000 rutas $/ \mathrm{km}^{2}$, mientras que para el ráster sin rangos llegan a ser superiores a 3000 rutas $/ \mathrm{km}^{2}$. Se obtiene un $59 \%$ de rutas altitudinales para el ráster con rangos y un $75.8 \%$ de rutas altitudinales para el ráster sin rangos. [Conclusiones]: Las modificaciones en el cálculo del ráster de costos provoca cambios en la red de rutas de conectividad, concentración de rutas en los sitios de conexión entre zonas o regiones, diferencias en los valores de la densidad de rutas y, finalmente, modificaciones en las condiciones altitudinales y longitudinales de las rutas.

Palabras claves: Áreas naturales protegidas; modelo costo-distancia; rutas de conectividad.

\section{Abstract}

[Introduction]: Most of the research dealing with connectivity route use cost-distance maps generated from the creation of ranges of the variables employed. [Objective]: This research evaluates the effect of the selected method for cost-distance map computation over the network of connectivity routes among viable vegetation patches in the protected areas of Granma province, Cuba. [Methodology]: The process generates two cost-distance raster (one with ranges and one without ranges) from which the connectivity routes are obtained. Additionally, the research includes the analysis of the effect of the selected method for raster computation over the connectivity route network. [Results]: The method identifies 3080 routes. Route density for raster with ranges reaches $1000 \mathrm{route} / \mathrm{km}^{2}$, while route density for raster without ranges reaches 3000 route $/ \mathrm{km}^{2}$. The $59 \%$ of the routes for the raster with ranges is

1 Centro Mexicano de Innovación en Energía-Océano, Instituto de Ciencias del Mar y Limnología, Universidad Nacional Autónoma de México, México. yandyro84@gmail.com; https://orcid.org/0000-0002-1792-6737

2 Posgrado en Geografía, Centro de Investigación en Geografía Ambiental, Universidad Nacional Autónoma de México, México. adonis. maikel@gmail.com; https://orcid.org/0000-0002-2515-2508

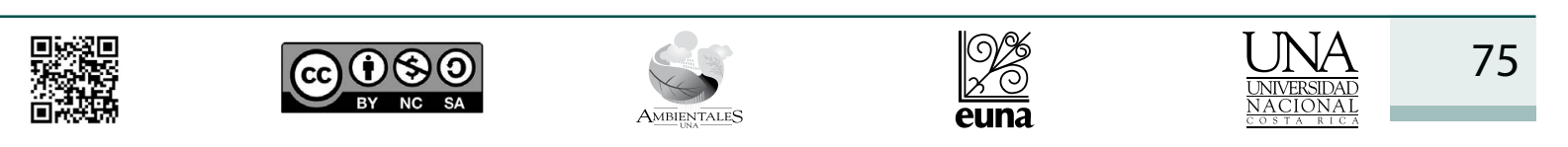




\section{Revista de CIENCIAS AMBIENTALES Tropical Journal of Environmental Sciences}

Revista de Ciencias Ambientales (Trop J Environ Sci)

e-ISSN: 2215-3896

(Enero-Junio, 2022). Vol 56(1): 75-95 DOI: https://doi.org/10.15359/rca.56-1.4 Open Access: www.revistas.una.ac.cr/ambientales e-mail: revista.ambientales@una.ac.cr Rodríguez Cueto Y., Ramón Puebla A.

altitudinal, while the $75.8 \%$ of the routes for the raster without ranges is altitudinal. [Conclusions]: The modification in the computation of the cost raster to displacement generates changes in the route network and concentration of the routes at the sites of connection between zones in the study area. Additionally, the values of route density change depending on the method of the cost raster computation, as well as the altitudinal condition of the routes.

Keywords: connectivity routes; cost-distance model; protect areas

\section{Introducción}

La progresiva maduración de los sistemas de protección de la naturaleza constatada a partir de los planteamientos de Johnstone et al. (2014) y Burkart et al. (2016) ha permitido, según Fahrig (2003) y Peled et al. (2016), que comience a considerarse en un número creciente de países la idea de establecer redes de corredores biológicos. La importancia trascendental de esta herramienta refiere Mindreau (2013), se aprecia en dos de las metas del Plan Estratégico para la Diversidad Biológica 2011-2020 del Convenio de Diversidad Biológica, que promueven la reducción significativa de la degradación y fragmentación de hábitats naturales, aplicando medidas de conservación eficaces a partir de la interconexión e integración de las áreas naturales protegidas (ANP) a paisajes más amplios.

Dicha interconexión e integración de las ANP parte de las premisas de Herrera Calvo y Díaz Varela (2013) referida a la existencia de fuertes relaciones entre los patrones del paisaje y las funciones y procesos ecológicos en este. Ello se fundamenta, para Bennett y Mulongoy (2006), en el supuesto de que los fragmentos conectados por un corredor de hábitat adecuado disminuyen la tasa de extinción y contribuyen a un mayor valor para la conservación que los hábitats aislados. En tal sentido, Fahrig (2003) y Bennett (2004) afirman que garantizar la conectividad entre "parches" de diferentes hábitats es importante, tanto para la dispersión de especies como para la protección de los propios hábitats.

A partir de estas aseveraciones, Bennett y Mulongoy (2006) y Chacón Salas (2016) argumentan que, en los estudios de los procesos ecológicos y de dispersión de las especies en un paisaje, es posible aplicar los modelos de conectividad para generar redes de conectividad estructural. Dichas redes se consideran una estructura espacial que pretende fungir como enlace entre ANP, cuya trayectoria está definida por las rutas que generan una menor resistencia al movimiento de especies. Dichas rutas se diseñan, a criterio de Bennett (2004) y Chacón Salas (2016), por modelos que producen escenarios gráficos sobre la permeabilidad del paisaje a la movilidad de los animales en función de la distancia máxima de dispersión ecológica, contra la resistencia ejercida por el uso del suelo.

Para realizar estudios de la conectividad a nivel de paisaje, se han desarrollado modelos matemáticos tanto teóricos como aplicados que permiten la solución de problemas específicos. Estos modelos simulan los flujos o desplazamientos en el paisaje (movimientos individuales, dinámica de meta-poblaciones), en tiempo continuo como los de Galpern (2013) y Johnstone et al. (2014); o en tiempo discreto, como los de Hanski (1994). Dichos modelos suelen centrase en

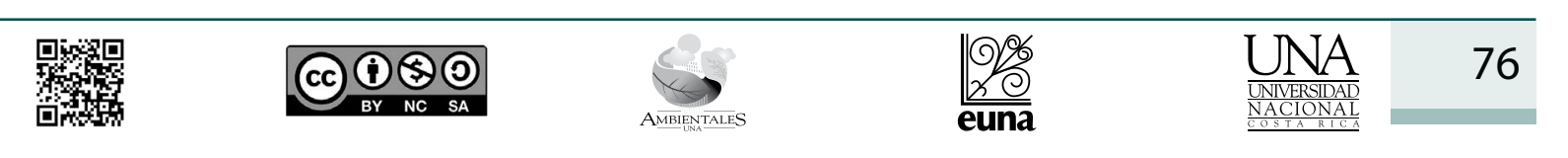




\section{Revista de CIENCIAS AMBIENTALES Tropical Journal of Environmental Sciences}

Revista de Ciencias Ambientales (Trop J Environ Sci)

e-ISSN: 2215-3896 (Enero-Junio, 2022). Vol 56(1): 75-95 DOI: https://doi.org/10.15359/rca.56-1.4 Open Access: www.revistas.una.ac.cr/ambientales e-mail: revista.ambientales@una.ac.cr Rodríguez Cueto Y., Ramón Puebla A.

un sólo tipo de hábitat o de elemento del paisaje para la elaboración de los mapas de fricción. Sin embargo, con el uso de los Sistemas de Información Geográfica (SIG), es posible integrar más variables en la elaboración de los modelos de simulación sin perder información referente a la funcionalidad de cada una de ellas, como se aprecia en los trabajos de Galpern (2013), Chacón Salas (2016), Colorado Zuluaga et al. (2017) y Ramón Puebla et al. (2020).

Actualmente, la metodología más utilizada para modelar rutas de conectividad (estructurales o funcionales) está basada en las superficies de resistencia que describen la conectividad del paisaje como su inverso a la resistencia al movimiento y a la dispersión de las especies, según Sawyer et al. (2011) y Zeller et al. (2012). Típicamente, estas superficies representan el grado en que el paisaje impide o facilita el movimiento de las especies. Al respecto, Galpern (2013) refiere su utilidad para representar la conectividad estructural entre dos puntos cualesquiera del paisaje como una distancia efectiva que se calcula a través de la ruta de menor costo en la superficie de resistencia que conecta dos puntos del paisaje.

La obtención de las superficies de resistencia al desplazamiento para la conectividad se determina por el tipo de cobertura, así como la presencia de elementos y actividades de carácter antrópico que se desarrollan en el territorio en los trabajos realizados por Chacón Salas (2016), Colorado Zuluaga et al. (2017) y Ramón Puebla et al. (2020). La dificultad más alta la imponen aquellas áreas donde la presencia de infraestructuras, cobertura existente y las actividades que se desarrollan se alejan de las condiciones naturales o constituyen un factor de estrés para la biota. La obtención de dichas superficies difiere en dos formas para generar el ráster final de costos. El primer método empleado por Arias et al. (2008), Céspedes et al. (2008), Colorado Zuluaga et al. (2017) y Ramón Puebla et al. (2020) genera las superficies de resistencia al desplazamiento, a partir de establecer un sistema de categorías para la cobertura del suelo y un sistema de rangos positivos o negativos preestablecidos por jerarquía y pesos para el resto de los componentes. El segundo método es empleado por Urban y Keitt (2001), Zeller et al. (2012), Galpern y Manseau (2013), y Chacón Salas (2016); y al igual que el anterior asigna los valores de resistencia al movimiento de las especies, dependiendo de la categoría de cobertura. Pero en este caso, las distancias a los elementos que puedan incidir positiva o negativamente ante la biota corresponden a variables continuas, sin el pre-establecimiento previo de rangos de distancia.

A partir de estas consideraciones, el objetivo general de esta investigación es evaluar las posibles diferencias en el trazado de las rutas de conectividad en las ANP de la provincia Granma, Cuba; a partir de la comparación de los dos métodos comúnmente más usados en la obtención del ráster de superficies de resistencia al desplazamiento. Esta comparativa de métodos se verá reflejada en la obtención de las potenciales rutas de conectividad entre los parches de bosque dentro de las ANP, y entre estas y los de las ANP cercanas, mediante un modelo de costo-distancia, con base en la elaboración del mapa de resistencia al desplazamiento entre los espacios núcleo de las ANP de la provincia Granma, Cuba.

En tal sentido, la investigación presentada es la primera de su tipo en Cuba, así como la primera que analiza las rutas de conectividad a partir de un modelo de ráster de costos sin

\begin{tabular}{|c|c|c|}
\hline 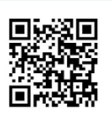 & (c) (1) (5) () & 77 \\
\hline
\end{tabular}




\section{Revista de CIENCIAS AMBIENTALES Tropical Journal of Environmental Sciences}

Revista de Ciencias Ambientales (Trop J Environ Sci)

e-ISSN: 2215-3896 (Enero-Junio, 2022) . Vol 56(1): 75-95 DOI: https://doi.org/10.15359/rca.56-1.4 Open Access: www.revistas.una.ac.cr/ambientales e-mail: revista.ambientales@una.ac.cr Rodríguez Cueto Y., Ramón Puebla A.

considerar la clasificación por rangos de las variables de entrada al modelo. Ello implica un paso de avance en el diseño de rutas de conectividad al mantener la complejidad estructural del espacio, complejidad que se reduce al reclasificar en rangos las variables de entrada en el modelo de generación del ráster de costos al desplazamiento.

\section{Metodología}

\section{1. Área de estudio}

Corresponde a la provincia Granma (ver Figura 1), localizada según los datos de Oficina Nacional de Estadísticas e Información (2019), en la parte sur oriental de Cuba, entre los 1949' 9.0" y los $20^{\circ} 45^{\prime} 43.8^{\prime \prime}$ de latitud norte, y los $76^{\circ} 11^{\prime} 45.7^{\prime \prime}$ y $77^{\circ} 44^{\prime} 27.9^{\prime \prime}$ de longitud oeste, con una superficie de $8362 \mathrm{~km}^{2}$ de extensión, lo que representa el 7.57 \% del área total de Cuba. La población es de 834 380 habitantes, para una densidad poblacional de $99.76 \mathrm{hab} . / \mathrm{km}^{2}$, considerada la quinta provincia más densamente poblada de Cuba. Limita al norte con las provincias de Las Tunas y Holguín, al este con las provincias de Holguín y Santiago de Cuba, al sur con la provincia Santiago de Cuba y el Mar Caribe y al oeste con el Golfo de Guacanayabo, con el cual comparte 260 km de litoral.

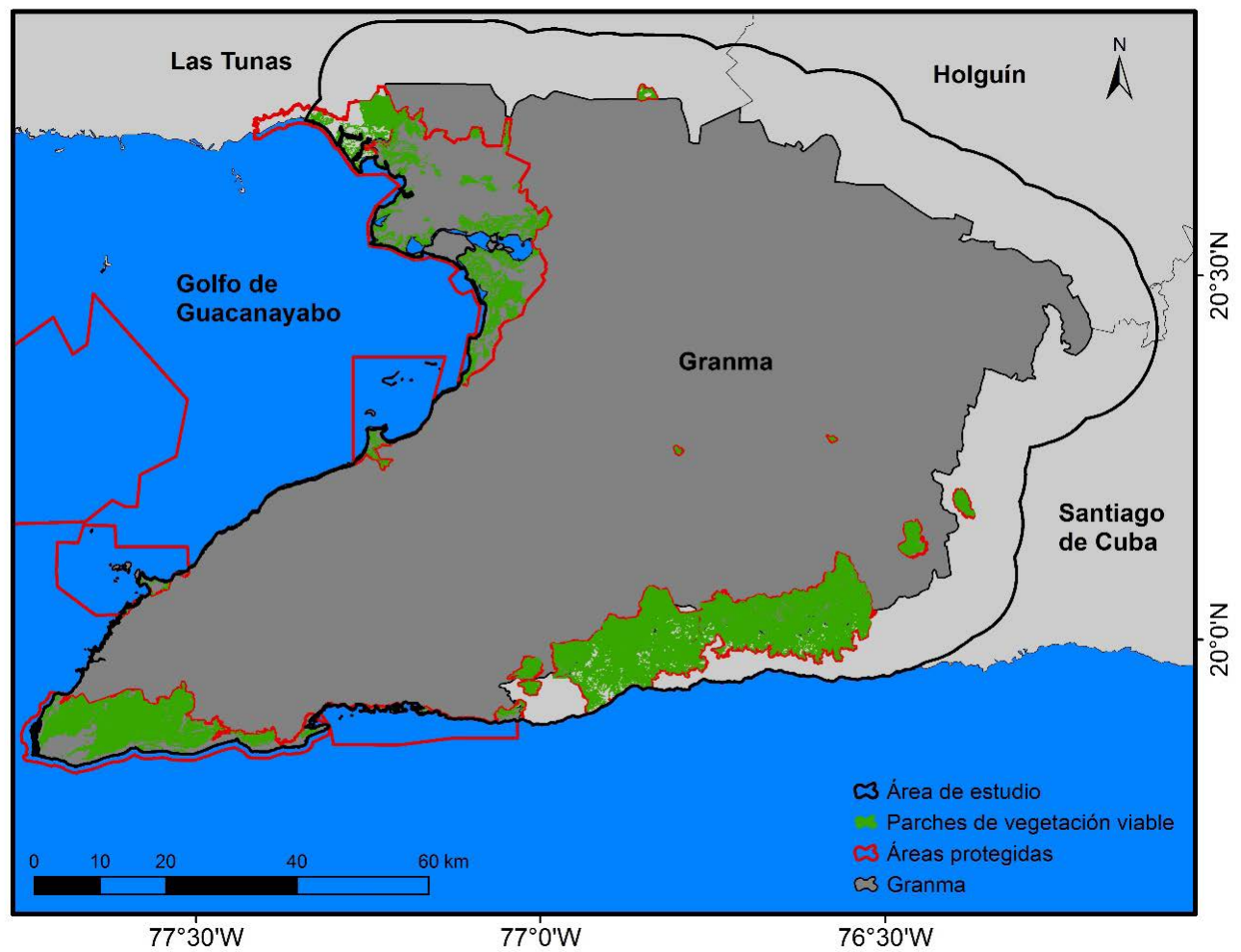

Figura 1. Situación geográfica de la provincia Granma.

Figure 1. Geographic situation of Granma province.

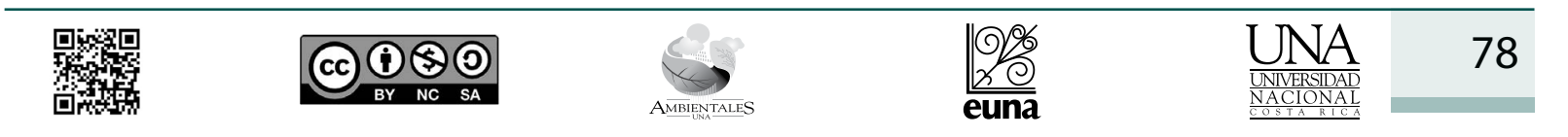




\section{Revista de CIENCIAS AMBIENTALES Tropical Journal of Environmental Sciences}

Revista de Ciencias Ambientales (Trop J Environ Sci)

e-ISSN: 2215-3896

(Enero-Junio, 2022) . Vol 56(1): 75-95

DOI: https://doi.org/10.15359/rca.56-1.4

Open Access: www.revistas.una.ac.cr/ambientales e-mail: revista.ambientales@una.ac.cr Rodríguez Cueto Y., Ramón Puebla A.

Esta región se divide en dos grandes morfoestructuras: las montañas de la Sierra Maestra, formado mayoritariamente por rocas vulcanógenas y vulcanógeno-sedimentarias; y la llanura Cauto-Guacanayabo, constituida principalmente por depósitos terrígeno-sedimentarios, principalmente aluviales y aluvio-marinos (Iturralde-Vinent, 2010).

El clima se caracteriza, según los datos de Oficina Nacional de Estadísticas e Información (2019), por la influencia marítima y de los vientos alisios del nordeste. Las precipitaciones son de carácter estacional con un promedio de 1600 milímetros, existiendo zonas de muy alta pluviosidad en la parte montañosa (valores superiores a los 2000 milímetros anuales) y zonas secas en la costa sur oriental (valores medios de 800 milímetros anuales). Estas características condicionan una cobertura vegetal diversa (González Alonso, 2012), en la que pueden encontrarse gran parte de las formaciones boscosas y complejos de vegetación presentes en el país. Además, se destaca una biota muy rica en endémicos vegetales y animales restringidos principalmente a los macizos montañosos y, a las costas bajas cenagosas, dando una destacada y particular distribución a los principales valores de diversidad biológica.

Toda esta riqueza natural y valores ecológicos, refiere Mateo (2017), están fuertemente impactados por los procesos de fragmentación y degradación del hábitat, provocados por las acciones antrópicas, a las que ha estado sometida la región por más de cuatro siglos. En dichas acciones predominó el uso irracional e intensivo, con prácticas agropecuarias y forestales inadecuadas; caracterizadas por una excesiva explotación de los recursos forestales y su extinción, para el establecimiento de cultivos varios, café, caña de azúcar y arroz, y el desarrollo ganadero, tanto en las zonas llanas como montañosas. A ello se vincula el posterior desarrollo de la urbanización y la infraestructura de comunicación entre la amplia red de asentamientos que evolucionó de forma paralela a este intenso proceso de asimilación socioeconómica del territorio.

No obstante, partiendo de los resultados del trabajo de Estrada et al. (2011), se puede señalar la existencia en la provincia Granma de áreas con vegetación natural y seminatural, hacia las zonas montañosas y costeras fundamentalmente. Dichas áreas, en su mayoría, componen hoy parte de los núcleos de conservación del sistema de ANP; con un claro predominio de bosques fragmentados y la carencia de un sistema de conectividad. Esta carencia de conectividad impone la necesidad del establecimiento de una red de hábitats intercomunicados que mantengan la conectividad de los procesos ecológicos a nivel de paisaje en esta provincia de Cuba.

\subsection{Modelo para el diseño de redes de conectividad ecológica}

Uno de los modelos más usados para el diseño de redes de conectividad es el propuesto por Céspedes et al. (2008), aplicado en Cuba, para la región de la Sierra Maestra por Ramón Puebla et al. (2020). Estos autores concuerdan en la existencia de tres componentes fundamentales para el diseño de dichas redes:

- Identificación de los fragmentos de bosques o áreas/nodos a conectar.

- Establecimiento de los niveles o grados de dificultad al desplazamiento entre los fragmentos de bosques o áreas/nodos a conectar.

\begin{tabular}{|c|c|c|c|c|c|}
\hline 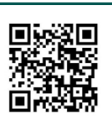 & (c) (i) (5) () & $\underset{\text { AMBENAIIES }}{80}$ & $\begin{array}{l}\frac{O \%}{2} \\
\frac{2}{\text { euna }} \\
\end{array}$ & 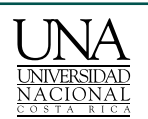 & 79 \\
\hline
\end{tabular}




\section{Revista de CIENCIAS AMBIENTALES Tropical Journal of Environmental Sciences}

Revista de Ciencias Ambientales (Trop J Environ Sci)

e-ISSN: 2215-3896

(Enero-Junio, 2022) . Vol 56(1): 75-95

DOI: https://doi.org/10.15359/rca.56-1.4

Open Access: www.revistas.una.ac.cr/ambientales e-mail: revista.ambientales@una.ac.cr Rodríguez Cueto Y., Ramón Puebla A.

- Modelación de la red de conectividad integrada por los fragmentos de bosques o áreas/ nodos, mediante el establecimiento de las rutas de menor dificultad al desplazamiento.

\subsubsection{Definición de las áreas núcleos o nodos de conectividad}

A efectos de este trabajo, se seleccionaron las ANP de la provincia con las categorías de manejo más estrictas, 11 en total, y con objetivos de manejo equivalentes a las categorías I y II de la UICN. También, se incluyeron en el análisis cuatro ANP correspondientes a las provincias colindantes, situadas a menos de $10 \mathrm{~km}$ de los límites de la provincia Granma y con similares categorías de manejo, dada la posibilidad de que exista algún tipo de conectividad entre estas y las ANP de Granma.

Un aspecto ciertamente novedoso en la investigación es la evaluación de la conectividad a partir de fragmentos de bosques considerados viables al interior de las ANP y no a partir de los bordes externos de estas, como tradicionalmente se hace. Esta decisión se tomó con base en que pueden existir fragmentos de coberturas al interior de las ANP, y cercano a los bordes de estas, que se correspondan con usos no factibles para garantizar la conectividad o el desplazamiento natural de las especies. Los aspectos considerados para declarar viable un fragmento de bosque se establecieron siguiendo criterios de autores como Kattan (2002) y se presentan en el Cuadro 1.

Cuadro 1. Criterios para que un parche de bosque sea considerado viable para el sostenimiento de comunidades y especies de fauna.

Table 1. Criteria for considering vegetation patch as viable for sustaining communities and fauna.

\begin{tabular}{|c|c|c|}
\hline Criterio & Planteamiento & Referencia \\
\hline Tamaño del hábitat & $\begin{array}{l}\text { Para este estudio se decidió tomar como viables los fragmentos iguales } \\
\text { o mayores a } 7 \text { ha. }\end{array}$ & Corredor Jiménez (2019) \\
\hline Efecto de borde & $\begin{array}{l}\text { Se tomó como criterio un ancho de borde de } 94 \mathrm{~m} \text {, considerando la } \\
\text { pérdida de la cobertura boscosa en la provincia Granma, según Del } \\
\text { Risco (1995). }\end{array}$ & Newmark (2001) \\
\hline Área interior absoluta & Áreas interiores superiores a 1 ha. & Hilty et al. (2006) \\
\hline
\end{tabular}

Fuente: elaboración propia, con base en Corredor Jiménez (2019), Newmark (2001) y Hilty et al. (2006).

\subsubsection{Criterios para establecer los niveles de dificultad para la conectividad y la superficie de fricción}

Se valoraron los criterios y experiencias de expertos internacionales y nacionales vertidos en los trabajos realizados por Arias et al. (2008), Céspedes et al. (2008), Chacón Salas (2016) y Ramón Puebla et al. (2020); así como los estudios sobre biodiversidad realizados en Cuba por Borroto-Páez y Mancina (2011), González Alonso (2012), González (2002) y Mancina y Cruz (2017), permitiendo identificar 5 factores fundamentales que, a juicio de los autores, pueden incidir en la permeabilidad del paisaje del territorio, los cuales son:

\begin{tabular}{|c|c|c|c|c|}
\hline 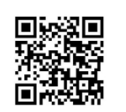 & (c) (1) (9) & $\underset{\text { AMBENTALES }}{\infty}$ & $\frac{\sigma \%}{\text { euna }}$ & 80 \\
\hline
\end{tabular}




\section{Revista de CIENCIAS AMBIENTALES Tropical Journal of Environmental Sciences}

Revista de Ciencias Ambientales (Trop J Environ Sci)
e-ISSN: 2215-3896
(Enero-Junio, 2022). Vol 56(1): 75-95
DOI: https://doi.org/10.15359/rca.56-1.4
Open Access: www.revistas.una.ac.cr/ambientales
e-mail: revista.ambientales@una.ac.cr
Rodríguez Cueto Y., Ramón Puebla A.

- Cobertura del suelo: las coberturas naturales y menos alteradas por las actividades humanas, que ofrecen la menor dificultad al movimiento de las especies (Bennet, 2004).

- Dinámica espacio-temporal de la cobertura del suelo: la permanencia de coberturas naturales y poco alteradas y los cambios positivos en las mismas favorecen la estabilidad espacio-temporal de las rutas de conectividad, de acuerdo con Céspedes et al. (2008); por lo que en este estudio se tuvo en cuenta el cambio de una cobertura alterada a una poco alterada y la presencia de coberturas favorables estables en el tiempo.

- Red hídrica: las zonas que se ubican cerca de la red hídrica ofrecen una menor dificultad para el movimiento de las especies, según Bennet (2004) y Arias et al. (2008). También se tuvo en cuenta la función de los grandes cuerpos de agua como barreras a la dispersión de las especies terrestres no aladas con escasa capacidad de nado, cuando exceden una longitud determinada. Para esta investigación, se tomó un ancho superior a los $30 \mathrm{~m}$.

- Asentamientos humanos: la presencia de estos son un obstáculo para el desplazamiento de algunas especies y se asume que los efectos negativos de los asentamientos para proporcionar conectividad en un paisaje disminuyen cuando aumenta la distancia entre una zona de tránsito y un poblado (Arias et al., 2008; Chacón Salas, 2016).

- Infraestructura vial: la presencia de infraestructura vial, refieren Arias et al. (2008) y Chacón Salas (2016), significa un obstáculo para el desplazamiento de las especies e incorpora en el paisaje perturbaciones asociadas al ruido. En este trabajo consideramos que los efectos negativos de la infraestructura vial disminuyen cuando aumenta la distancia entre una zona en el paisaje y este tipo de infraestructura.

Para la evaluación de estos criterios y generar el mapa de fricción, por el método de los rangos, se establecieron rangos y pesos para ponderar su comportamiento al desplazamiento de las especies en el territorio. Dichos rangos y pesos se determinaron con base en la escala ordinal y método de clasificación ya usado por Ramón Puebla et al. (2020). Los criterios de clasificación obtenidos a partir de este método, la jerarquía, el peso y la ponderación de cada componente, se presentan en el Cuadro 2.

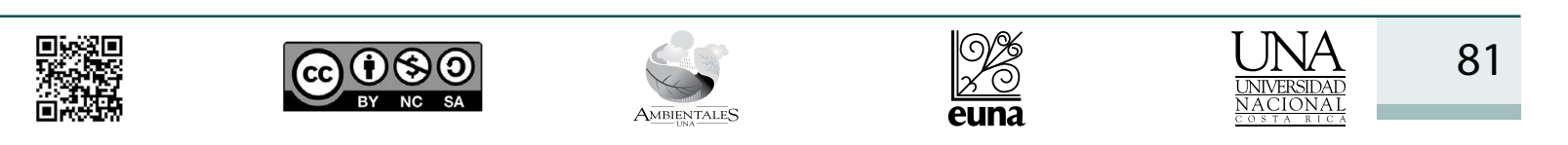




\section{Revista de CIENCIAS AMBIENTALES Tropical Journal of Environmental Sciences}

Revista de Ciencias Ambientales (Trop J Environ Sci)

e-ISSN: 2215-3896

(Enero-Junio, 2022). Vol 56(1): 75-95 DOI: https://doi.org/10.15359/rca.56-1.4 Open Access: www.revistas.una.ac.cr/ambientales e-mail: revista.ambientales@una.ac.cr Rodríguez Cueto Y., Ramón Puebla A.

Cuadro 2. Categorías de dificultad al desplazamiento establecida para cada clase.

Table 2. Category of difficulty for displacement established for every class.

\begin{tabular}{|c|c|c|c|c|c|c|c|}
\hline \multirow{2}{*}{ Componente } & \multicolumn{5}{|c|}{ Categorías de dificultad al desplazamiento } & \multirow[t]{2}{*}{ Jerarquía } & \multirow[t]{2}{*}{ Peso } \\
\hline & 1 & 2 & 3 & 4 & 5 & & \\
\hline $\begin{array}{l}\text { Cobertura del } \\
\text { suelo }\end{array}$ & $\begin{array}{l}\text { Bosques y } \\
\text { humedales }\end{array}$ & Matorrales & $\begin{array}{l}\text { Herbazales y } \\
\text { pastizales }\end{array}$ & $\begin{array}{l}\text { Áreas de } \\
\text { cultivos }\end{array}$ & $\begin{array}{l}\text { Superficies } \\
\text { desnudas y } \\
\text { cuerpos de } \\
\text { agua }\end{array}$ & 1 & 0.4 \\
\hline $\begin{array}{l}\text { Dinámica } \\
\text { espacio- } \\
\text { temporal de } \\
\text { la cobertura } \\
\text { boscosa }\end{array}$ & $\begin{array}{l}\text { Mantiene la } \\
\text { cobertura de } \\
\text { bosques y } \\
\text { humedales o } \\
\text { cambia a estas }\end{array}$ & $\begin{array}{l}\text { Mantiene la } \\
\text { cobertura de } \\
\text { matorrales o } \\
\text { cambia este }\end{array}$ & $\begin{array}{l}\text { Mantiene la } \\
\text { cobertura de } \\
\text { herbazales y } \\
\text { pastizales o } \\
\text { cambia a estos }\end{array}$ & $\begin{array}{l}\text { Mantiene la } \\
\text { cobertura } \\
\text { de áreas } \\
\text { cultivables } \\
\text { o cambia a } \\
\text { estos }\end{array}$ & $\begin{array}{l}\text { Se mantiene } \\
\text { la superficie } \\
\text { desnuda, o } \\
\text { cambia a estas }\end{array}$ & 2 & 0.2 \\
\hline $\begin{array}{l}\text { Distancia a la } \\
\text { red fluvial (m) }\end{array}$ & menos de 30 & de 31 a 60 & de 61 a 100 & de 101 a 200 & mayor a 200 & 2 & 0.2 \\
\hline $\begin{array}{l}\text { Distancia a los } \\
\text { asentamientos } \\
\text { humanos }(\mathrm{m})\end{array}$ & mayor a 1001 & de 501 a 1000 & de 201 a 500 & de 51 a 200 & menos de 50 & 3 & 0.1 \\
\hline $\begin{array}{l}\text { Distancia a } \\
\text { infraestructura } \\
\text { vial }(\mathrm{m})\end{array}$ & mayor a 1001 & de 501 a 1000 & de 201 a 500 & de 51 a 200 & menos de 50 & 3 & 0.1 \\
\hline
\end{tabular}

Fuente: elaboración propia.

Para la realización de este trabajo se identificaron las coberturas del suelo para los años 2000 y 2020, de acuerdo con Cheng et al. (2014). Una vez establecidas las coberturas, se identificaron las zonas de dinámica, tanto aquellas que cambian hacia un tipo de cobertura favorable a la conectividad como las que permanecen estables en el periodo.

En paralelo, se realizó la reclasificación de las capas correspondientes a los demás criterios, mediante el empleo de la herramienta Euclidean Distance, para calcular un ráster de distancias a cada uno de los elementos geométricos existentes en las capas de cada criterio o variable analizada. Una vez que los ráster estuvieron elaborados, con el uso de la herramienta Weighted Sum se elaboró el ráster de fricción y, mediante el empleo de la herramienta Cost Distance, se obtuvo el ráster de los costos al desplazamiento.

Finalmente, con la capa de los parches de vegetación natural que cumplieron los requerimientos establecidos para ser considerados viables dentro de cada ANP y el ráster de costos, se realizó el procedimiento ilustrado en la Figura 2, a partir de un proceso desarrollado en un ambiente de Model Builder de ArcMap, en su versión 10.4.

\begin{tabular}{|c|c|c|c|c|}
\hline 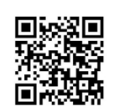 & (c) (1) (9) & $\underset{A M B E \text { INTALIES }}{\infty}$ & $\frac{9 \%}{\text { euna }}$ & 82 \\
\hline
\end{tabular}




\section{Revista de CIENCIAS AMBIENTALES Tropical Journal of Environmental Sciences}

Revista de Ciencias Ambientales (Trop J Environ Sci)

e-ISSN: 2215-3896

(Enero-Junio, 2022). Vol 56(1): 75-95 DOI: https://doi.org/10.15359/rca.56-1.4 Open Access: www.revistas.una.ac.cr/ambientales e-mail: revista.ambientales@una.ac.cr Rodríguez Cueto Y., Ramón Puebla A.

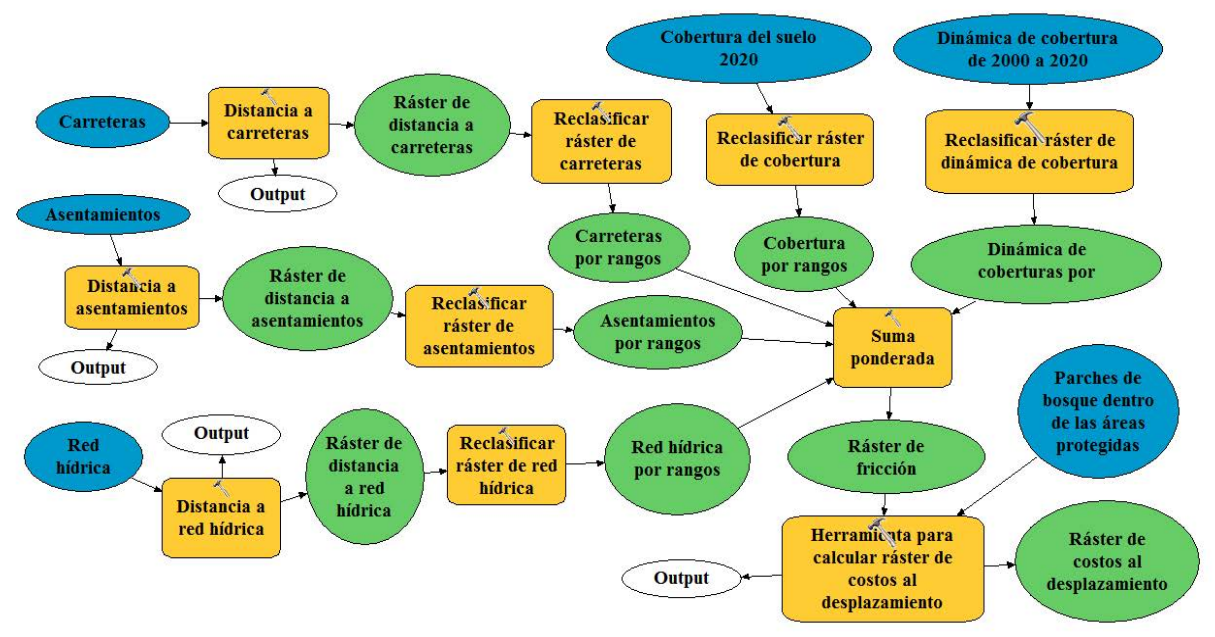

Figura 2. Representación del modelo para el cálculo del ráster de costo al desplazamiento con rangos y ponderaciones.

Figure 2. Representation of the model for computing the cost raster with ranged and weighted values.

Para el cálculo del ráster de costos al desplazamiento sin rangos, se introdujo un cambio fundamental: la eliminación del paso de reclasificar los componentes de barrera en rangos. Esto se debe al uso de los valores de distancia a los elementos de barrera directamente. En el caso de las carreteras y asentamientos, se consideró que mientras más alejado de estos elementos más factible es la conectividad. Por lo tanto, cada ráster de distancia se restó del máximo valor de distancia correspondiente a este, para obtener el inverso de esas distancias. Para el caso de la red hídrica, se consideró que la presencia de estos elementos es una barrera, por lo que se hizo una reclasificación de 0 para los píxeles con presencia de agua y 10 para los píxeles sin presencia de agua. En la Figura 3 se ilustra el proceso desarrollado en un ambiente de Model Builder de ArcMap, en su versión 10.4.

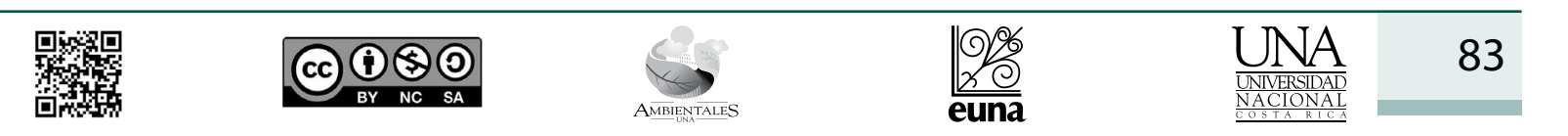




\section{Revista de CIENCIAS AMBIENTALES Tropical Journal of Environmental Sciences}

Revista de Ciencias Ambientales (Trop J Environ Sci)

e-ISSN: 2215-3896

(Enero-Junio, 2022). Vol 56(1): 75-95 DOI: https://doi.org/10.15359/rca.56-1.4 Open Access: www.revistas.una.ac.cr/ambientales e-mail: revista.ambientales@una.ac.cr Rodríguez Cueto Y., Ramón Puebla A.

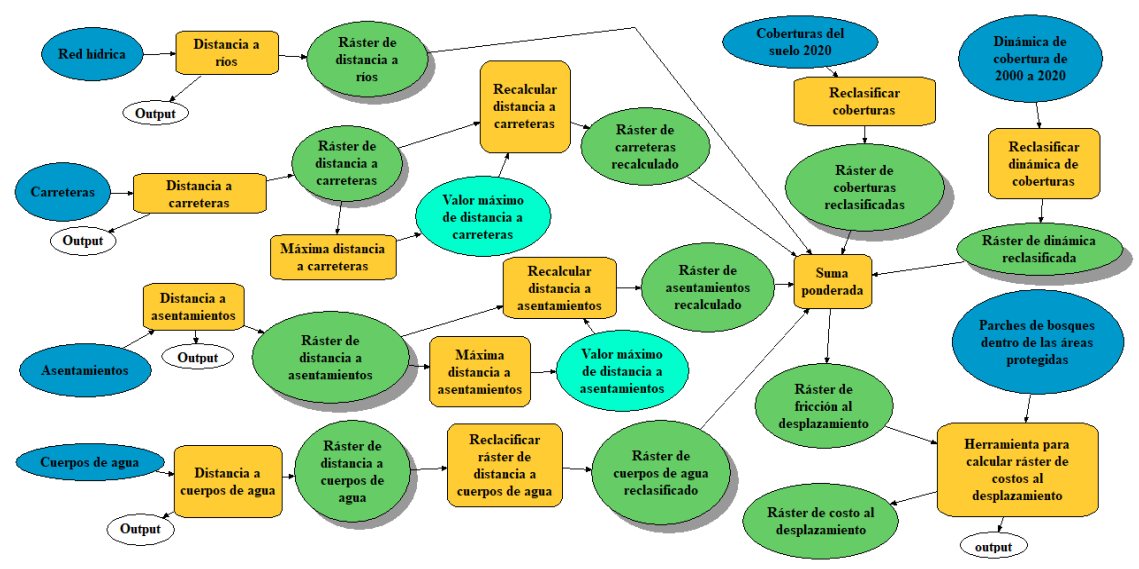

Figura 3. Representación del modelo para el cálculo del raster de costo al desplazamiento sin rangos.

Figure 3. Representation of the model for computing the cost raster without ranges.

\subsubsection{Modelación de las redes de conectividad ecológica}

La modelación de la red de conectividad se realiza mediante un modelo de costo-distancia, empleando funciones de Python, versión 3.6, sobre la interface de PyCharm, en su versión 2020.1. Este identifica las rutas de conectividad entre los centroides de los fragmentos de bosques (nodos) sobre el mapa de resistencia al desplazamiento. Utilizando cada nodo como punto de origen a conectar sobre la superficie de costos creada, se generan redes de conectividad desde el nodo de origen hacia los nodos de destino por las rutas con menor dificultad al desplazamiento. Esto implica que la ruta diseñada se corresponda con aquella que posee el menor valor acumulado de desplazamiento sobre el ráster de costos. Una vez obtenidas las rutas, se elaboró el ráster de densidad de rutas con la herramienta Line Density. Posteriormente, se calcularon las estadísticas para cada ANP, con el empleo de las herramientas Tabulate intersection y Summary Statistics.

$\mathrm{El}$ análisis de las condiciones altitudinales y longitudinales de las rutas identificadas se realizó con base en los pisos altimétricos de la vegetación, según Capote y Berazain (1984), para evaluar el rango en función de las fajas que conforman los ecosistemas de la provincia, los cuales se correspondieron con los siguientes rangos: $0 \mathrm{~m}-150 \mathrm{~m} ; 151 \mathrm{~m}-450 \mathrm{~m} ; 451 \mathrm{~m}-550 \mathrm{~m} ; 551$ $\mathrm{m}-850 \mathrm{~m} ; 851 \mathrm{~m}-1000 \mathrm{~m}$ y mayor de $1001 \mathrm{~m}$. Una vez obtenidas las rutas, se realizaron los perfiles topográficos y se identificaron los valores máximos y mínimos de altura. Dichos valores se compararon con los pisos altitudinales anteriormente definidos, mediante el empleo de diferentes funciones de Python.

\begin{tabular}{|c|c|c|c|c|c|}
\hline 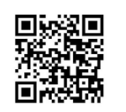 & (c) (1) () (2) & $\underset{\text { AMBENENALIES }}{8}$ & $\frac{1 \%}{\text { euna }}$ & 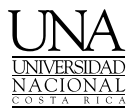 & 84 \\
\hline
\end{tabular}




\section{Revista de CIENCIAS AMBIENTALES Tropical Journal of Environmental Sciences}

Revista de Ciencias Ambientales (Trop J Environ Sci)

e-ISSN: 2215-3896 (Enero-Junio, 2022) . Vol 56(1): 75-95 DOI: https://doi.org/10.15359/rca.56-1.4 Open Access: www.revistas.una.ac.cr/ambientales e-mail: revista.ambientales@una.ac.cr Rodríguez Cueto Y., Ramón Puebla A.

\section{Resultados y discusión}

\subsection{Identificación de elementos de origen-destino}

El proceso de identificación de los sitios para la creación de las rutas de conectividad permitió identificar 56 parches de vegetación natural que cumplen con los requisitos necesarios para garantizar la conectividad y conservación. Dichos parches se convierten en los nodos de origen y destino de las rutas (ver Figura 4). Además, estos coinciden en la zona sur del área de estudio y a nivel de áreas protegidas, con las identificadas por Ramón Puebla et al. (2020) para el diseño de propuestas de rutas de conectividad en la Sierra Maestra, Cuba.

El Cuadro 3 muestra la distribución de parches y nodos por ANP, donde llama la atención que el $32 \%$ de los nodos se concentran en una sola ANP, Delta del Cauto; mientras que ocho (8) ANP presentan solamente un (1) nodo derivado de parches de vegetación natural viable para la conectividad. En cinco de esas ocho ANP con un único nodo, los parches correspondientes a esos nodos ocupan más del $90 \%$ del territorio del ANP, lo que resalta la importancia de los parches de vegetación en la conectividad de dichas ANP, al ser el único parche de vegetación viable a la conectividad.

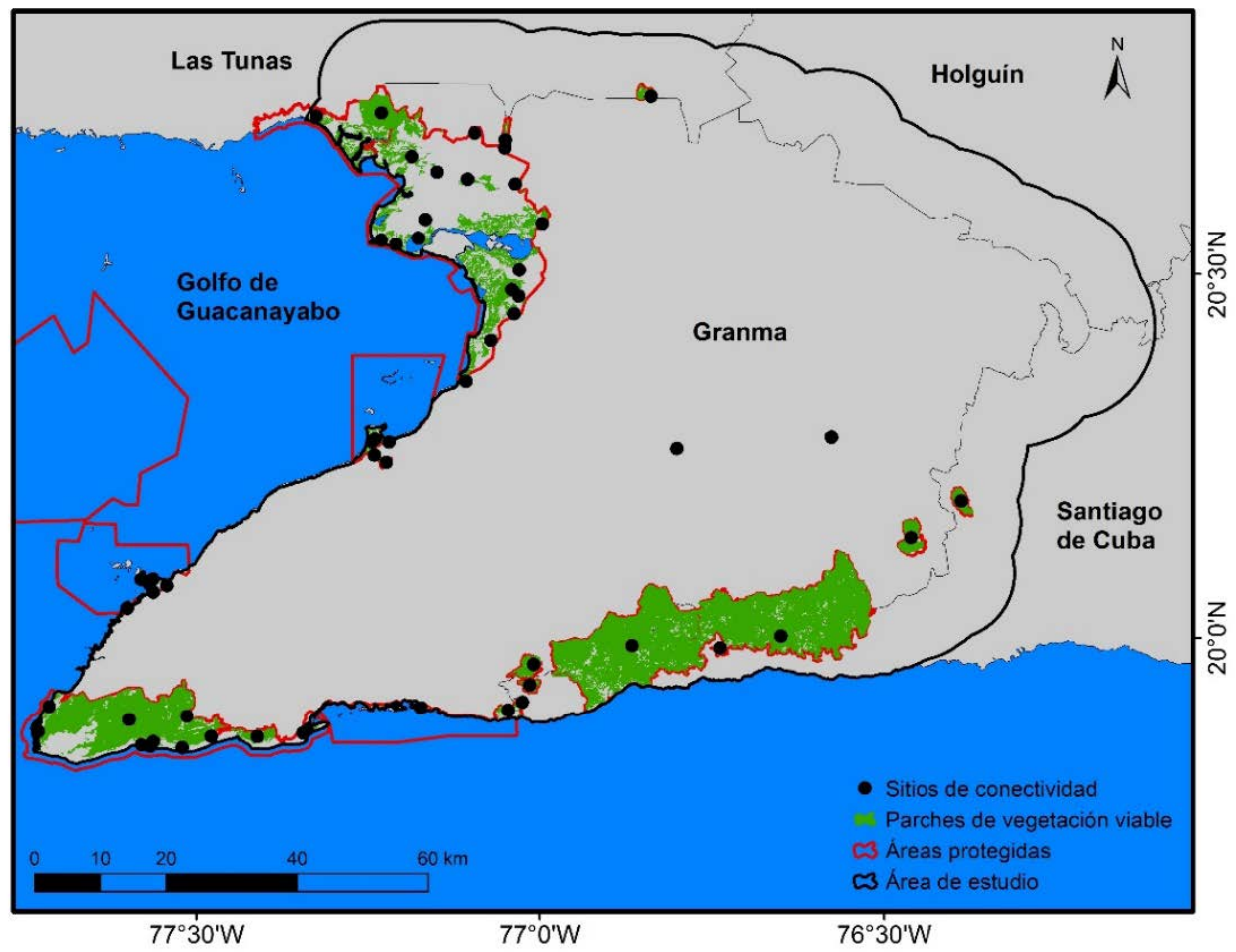

Figura 4. Nodos de origen-destinos identificados a partir de los parches de vegetación viables dentro de las ANP seleccionadas. Fuente: Centro Nacional de Áreas Protegidas (Globelandcover30, 2021)

Figure 4. Origin-destiny nodes identified from viable patches of vegetation inside the selected protected areas. Source: Protected Areas National Centre (Globelandcover30, 2021)

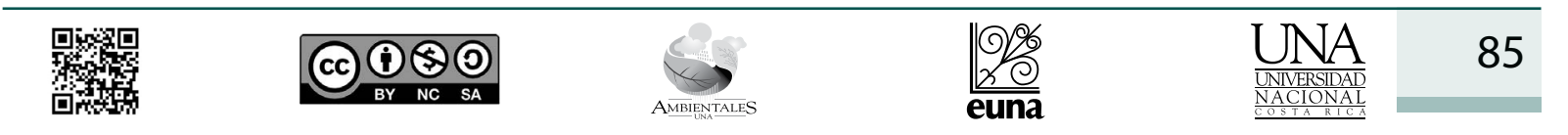




\section{Revista de CIENCIAS AMBIENTALES Tropical Journal of Environmental Sciences}

Revista de Ciencias Ambientales (Trop J Environ Sci)

e-ISSN: 2215-3896

(Enero-Junio, 2022) . Vol 56(1): 75-95 DOI: https://doi.org/10.15359/rca.56-1.4 Open Access: www.revistas.una.ac.cr/ambientales e-mail: revista.ambientales@una.ac.cr Rodríguez Cueto Y., Ramón Puebla A.

En tal sentido, Bennett (2004) señala que la mayor cantidad de parches aumenta la fragmentación, por lo que aquellas ANP con mayor cantidad de parches o nodos de conectividad están más fragmentadas y el desplazamiento dentro de ellas es más difícil para las especies.

Cuadro 3. Cantidad de parches de vegetación natural y nodos generados en las ANP.

Table 3. Patches and nodes corresponding to each protected area.

\begin{tabular}{clcccc}
\hline No & \multicolumn{1}{c}{ ANP } & Parches & Área $\left(\mathbf{k m}^{2}\right)$ & \% del área total & Nodos \\
\hline 1 & Banco de Buena Esperanza-Managuano & 8 & 3.85 & 0.43 & 6 \\
2 & Caraquitas $^{+}$ & 1 & 4.20 & 96.86 & 1 \\
3 & Delta del Cauto & 33 & 142.54 & 24.57 & 18 \\
4 & Desembarco del Granma & 18 & 180.04 & 55.20 & 12 \\
5 & El Gigante & 1 & 12.22 & 86.59 & 1 \\
6 & El Macío & 11 & 5.51 & 3.85 & 3 \\
7 & Ensenada de Gua y Cayos de Manzanillo & 6 & 7.72 & 4.67 & 5 \\
8 & Monte Naranjito & 1 & 3.40 & 79.81 & 1 \\
9 & Monte Natural Cupaynicú & 1 & 0.50 & 86.34 & 1 \\
10 & Monte Palmarito & 1 & 0.87 & 93.07 & 1 \\
11 & Ojo de Agua-Monte Cabaniguán $^{+}$ & 5 & 41.92 & 40.10 & 2 \\
12 & Pico Bayamesa & 2 & 225.08 & 92.99 & 2 \\
13 & Pico Caracas & 2 & 8.31 & 86.03 & 1 \\
14 & Pozo Prieto & 1 & 7.00 & 94.07 & 1 \\
15 & Turquino & 3 & 207.38 & 91.56 & 1 \\
& & $94^{*}$ & $850.5^{*}$ & $62.4^{* *}$ & $56^{*}$ \\
\hline
\end{tabular}

+ ANP en las provincias colindantes. ${ }^{\star}$ Suma de los valores. ${ }^{\star \star}$ Promedio de los valores

\subsection{Generación de los ráster de costos al desplazamiento}

La identificación o generación de los ráster de costos al desplazamiento se realizó de dos formas, tal y como se explica en el acápite metodológico. Primeramente, se generó un ráster de costos por rangos, para luego generar un ráster de costos al desplazamiento que no incluyera rangos y que usa los valores reales de las variables ponderadas con su peso relativo dentro del análisis.

Aun cuando para ambos métodos de generación del ráster de costo al desplazamiento (Figura 5), se presentan altos valores en la zona nordeste del territorio, hay diferencias que señalar. La creación del ráster de costos mediante el método con rangos (Figura 5a) genera una zona de continuidad entre dicha área nordeste y el centro de la provincia, con valores de costo de medio a alto; mientras que en el ráster sin rangos (Figura 5b) esa zona de continuidad presenta valores de costos de bajo a medio, lo que favorecería la generación de rutas a través de esa área. Adicionalmente, la superficie con valores de costo cercanos a medio en la zona suroccidental del área de estudio es mayor en el ráster de costo con rangos (Figura 5a) que en el ráster de costos sin rangos (Figura 5b). El ráster de costos con rangos (Figura 5a) genera una especie de línea continua de valores medios que une el centro del área de estudio con la zona suroccidental de esta,

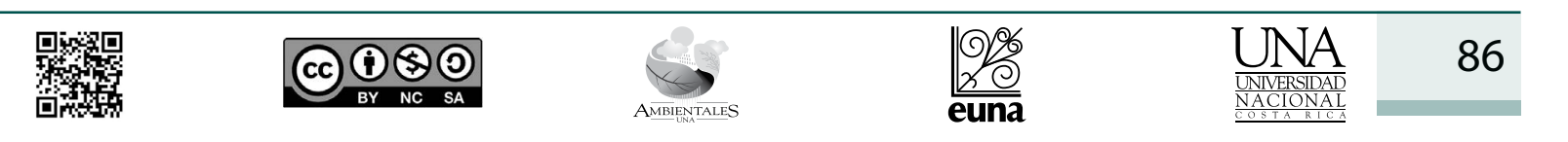




\section{Revista de CIENCIAS AMBIENTALES Tropical Journal of Environmental Sciences}

Revista de Ciencias Ambientales (Trop J Environ Sci)

e-ISSN: 2215-3896 (Enero-Junio, 2022) . Vol 56(1): 75-95 DOI: https://doi.org/10.15359/rca.56-1.4 Open Access: www.revistas.una.ac.cr/ambientales e-mail: revista.ambientales@una.ac.cr Rodríguez Cueto Y., Ramón Puebla A.

lo que pudiera generar restricciones a la conectividad entre la costa occidental y la costa sur; y no ocurre en el ráster de costos sin rangos (Figura 5b). Finalmente, el último cambio llamativo está relacionado con la zona noroccidental del territorio, en la desembocadura del río Cauto, donde en el ráster de costos al desplazamiento con rangos (Figura 5a), se observa que aparece con valores cercanos a bajo, mientras que en el ráster de costos al desplazamiento sin rangos (Figura 5b) se observa que aparecen regiones con valores cercanos a medio, lo que probablemente influirá en la creación de las rutas de conectividad.

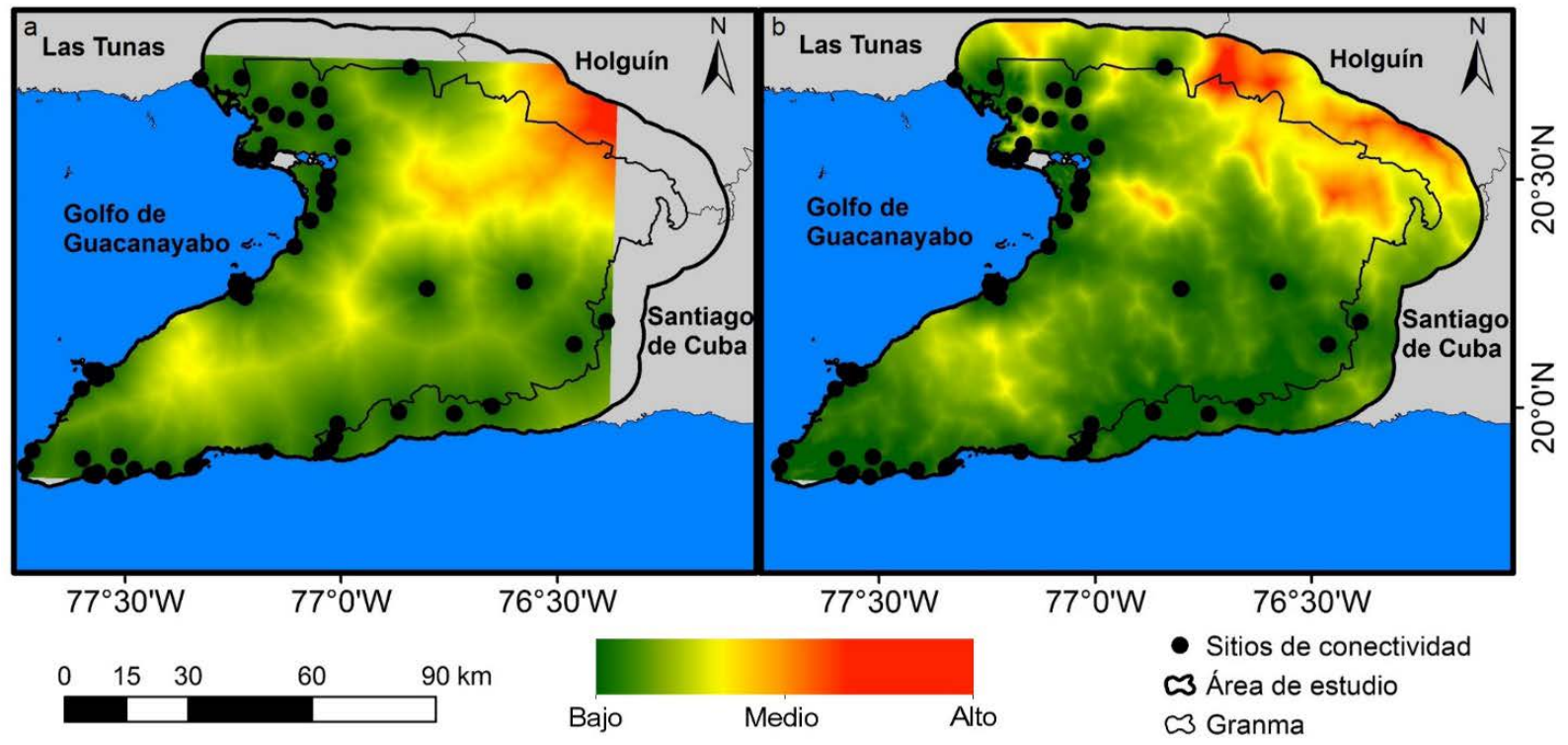

Figura 5. Ráster de costos al desplazamiento para ambos métodos. a) con la presencia de rangos, b) sin la presencia de rangos.

Figure 5. Cost raster to displacement for both methods. a) with the presence of ranges, b) without the presence of ranges.

Las distribuciones de los valores de costos al desplazamiento obtenidos por ambos métodos son similares a los obtenidos por Ramón Puebla et al. (2020), con valores entre bajo y medio, más cercanos a medio, en la zona de coincidencia de dicho trabajo y el que se presenta en esta investigación. Dichas zonas de coincidencia se presentan hacia el centro del área de estudio, lo que sería la periferia de la Sierra Maestra, área analizada en Ramón Puebla et al. (2020).

\subsection{Comparación de métodos de generación de ráster de costos.}

La comparación de los métodos de generación de los ráster de costos se centra en aspectos como: el trazado de las rutas de conectividad, la densidad de rutas a partir del trazado de estas y las condiciones altitudinales y longitudinales de las rutas identificadas en cada método empleado.

\begin{tabular}{|c|c|c|}
\hline 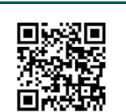 & (c) $\underset{\mathrm{BY}}{(\mathrm{i})(\mathrm{NC}} \mathrm{SA}_{\mathrm{SA}}$ & 87 \\
\hline
\end{tabular}




\section{Revista de CIENCIAS AMBIENTALES Tropical Journal of Environmental Sciences}

Revista de Ciencias Ambientales (Trop J Environ Sci)

e-ISSN: 2215-3896 (Enero-Junio, 2022) . Vol 56(1): 75-95 DOI: https://doi.org/10.15359/rca.56-1.4 Open Access: www.revistas.una.ac.cr/ambientales e-mail: revista.ambientales@una.ac.cr Rodríguez Cueto Y., Ramón Puebla A.

\subsubsection{Trazado de las rutas de conectividad}

El método de diseño del ráster de costos al desplazamiento influye directamente en el trazado de las rutas de conectividad, lo que se percibe, en primer lugar, por la simplificación de la red de rutas de conectividad, la concentración de rutas en ciertas áreas y la desaparición de rutas en otras. Este proceso de simplificación, concentración y desaparición es evidente entre la parte oeste de la provincia (costa del Golfo de Guacanayabo) y el nodo ubicado al norte y la zona al centro y sur de la provincia. Hacia el nodo al norte, de 3 rutas con el raster de costos con rangos (Figura 6a), se modifica a una con el raster de costos sin rangos (Figura 6b); mientras que, al centro y sur del área, de 2 zonas de rutas en el ráster de costos con rangos (Figura 6a) se obtiene solamente una zona en el ráster de costos sin rangos (Figura 6b). En segundo lugar, se percibe una mayor complejidad de rutas al interior de los parches de vegetación viable con el empleo del ráster de costos sin rangos (Figura 6b); mientras que el uso del ráster de costos con rangos (Figura 6a) simplifica las rutas al interior de los parches de vegetación viable.

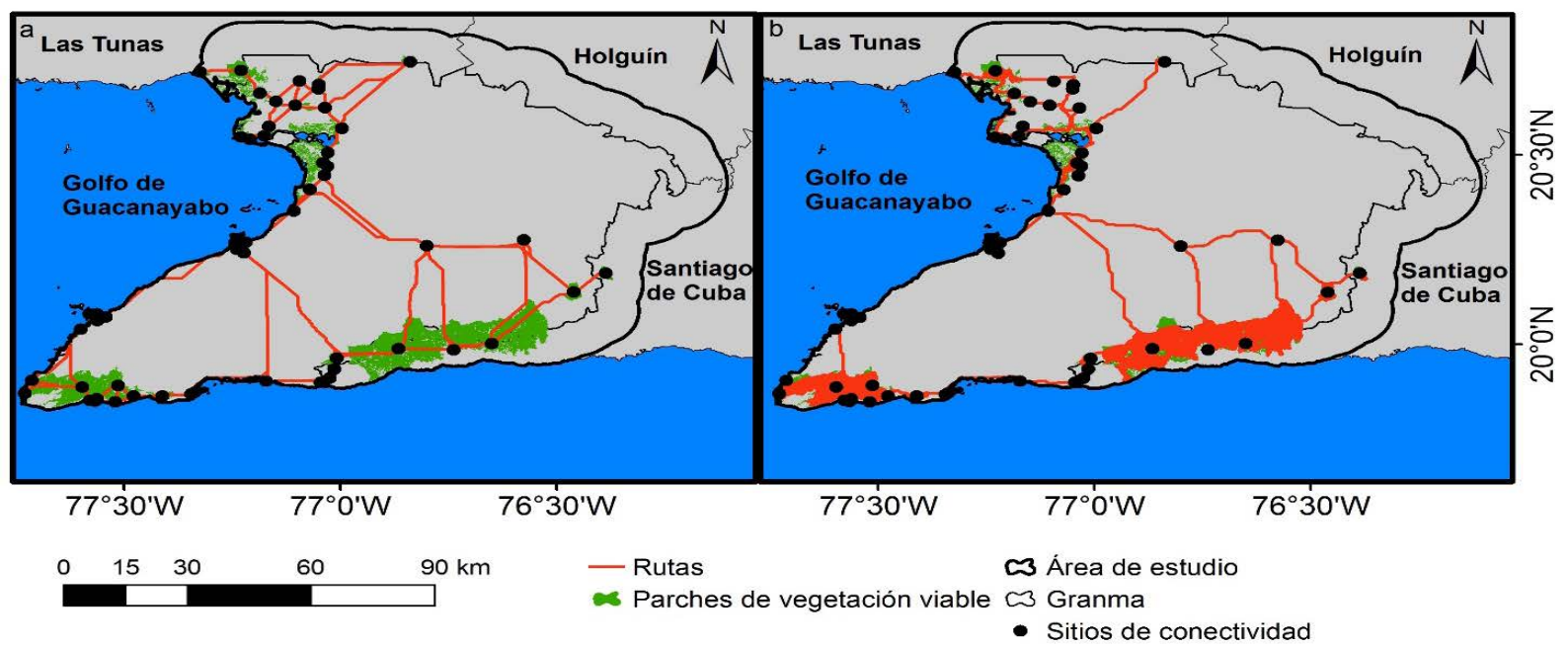

Figura 6. Rutas de conectividad identificadas. a) con el empleo del ráster de costos con rangos, b) con el empleo del ráster de costos sin rangos.

Figure 6. Connectivity routes identified. a) Using the cost raster with ranges, b) Using the cost raster without ranges.

Estos cambios en el trazado de las rutas de conectividad implican una reducción en la posibilidad de movimiento de las especies de un sitio a otro, aumentando el nivel de importancia para la conectividad en ciertos sitios y zonas y concentrando la conectividad en determinadas zonas del área de estudio. Adicionalmente, las zonas donde para ambos métodos coinciden las rutas de conectividad son zonas sumamente útiles para la gestión territorial focalizada en la conservación, de acuerdo con Colorado Zuluaga et al. (2017). En tal sentido, la costa centro-este así como la zona central y la costa sur del área de estudio son regiones muy importantes para la

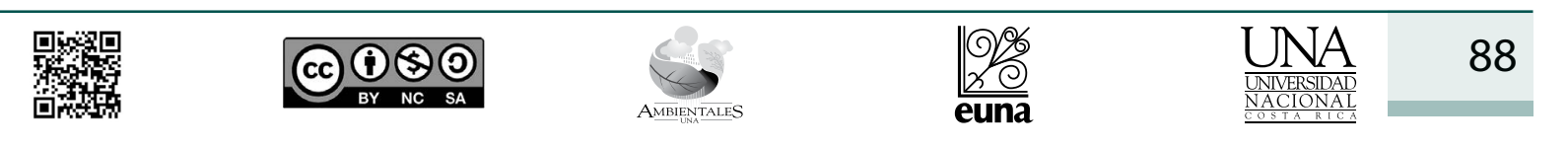




\section{Revista de CIENCIAS AMBIENTALES Tropical Journal of Environmental Sciences}

Revista de Ciencias Ambientales (Trop J Environ Sci) e-ISSN: 2215-3896 (Enero-Junio, 2022) . Vol 56(1): 75-95 DOI: https://doi.org/10.15359/rca.56-1.4 Open Access: www.revistas.una.ac.cr/ambientales e-mail: revista.ambientales@una.ac.cr Rodríguez Cueto Y., Ramón Puebla A.

conservación y la conectividad, puesto que poseen rutas de conectividad para ambos métodos de obtención del ráster de costos al desplazamiento.

\subsubsection{Densidad de rutas de conectividad}

Las modificaciones anteriormente mencionadas tienen un efecto directo sobre la densidad de rutas de conectividad en el área de estudio. Las diferencias se observan en la Figura 7, a continuación, donde es evidente que el empleo del ráster de costos con rangos (Figura 7a) genera menores valores de número de rutas por kilómetro cuadrado que el ráster de costos sin rangos (Figura $7 \mathbf{b}$ ), además de modificarse las zonas con mayores valores de densidad de rutas.

Los máximos valores de densidad de rutas en el ráster de costos con rangos (Figura 7a) son más de tres veces inferiores a los máximos valores de densidad de rutas en el ráster de costos sin rangos (Figura $\mathbf{7 b}$ ); además, la localización de dichos valores máximos cambia de un ráster de costo a otro. Mientras en el ráster de costo con rangos (Figura 7a) los máximos valores se localizan al centro y oeste del área, sobre las propias rutas; en el ráster de costos sin rangos (Figura 7b), los máximos valores se localizan al interior de las ANP. No obstante, la mayor parte del área de estudio presenta valores de densidad inferiores a las 25 rutas $/ \mathrm{km}^{2}$ con el empleo de ambos métodos de cálculo del ráster de costos, lo que muestra, en cierta medida, la concentración de las rutas en zonas específicas por ambos métodos.

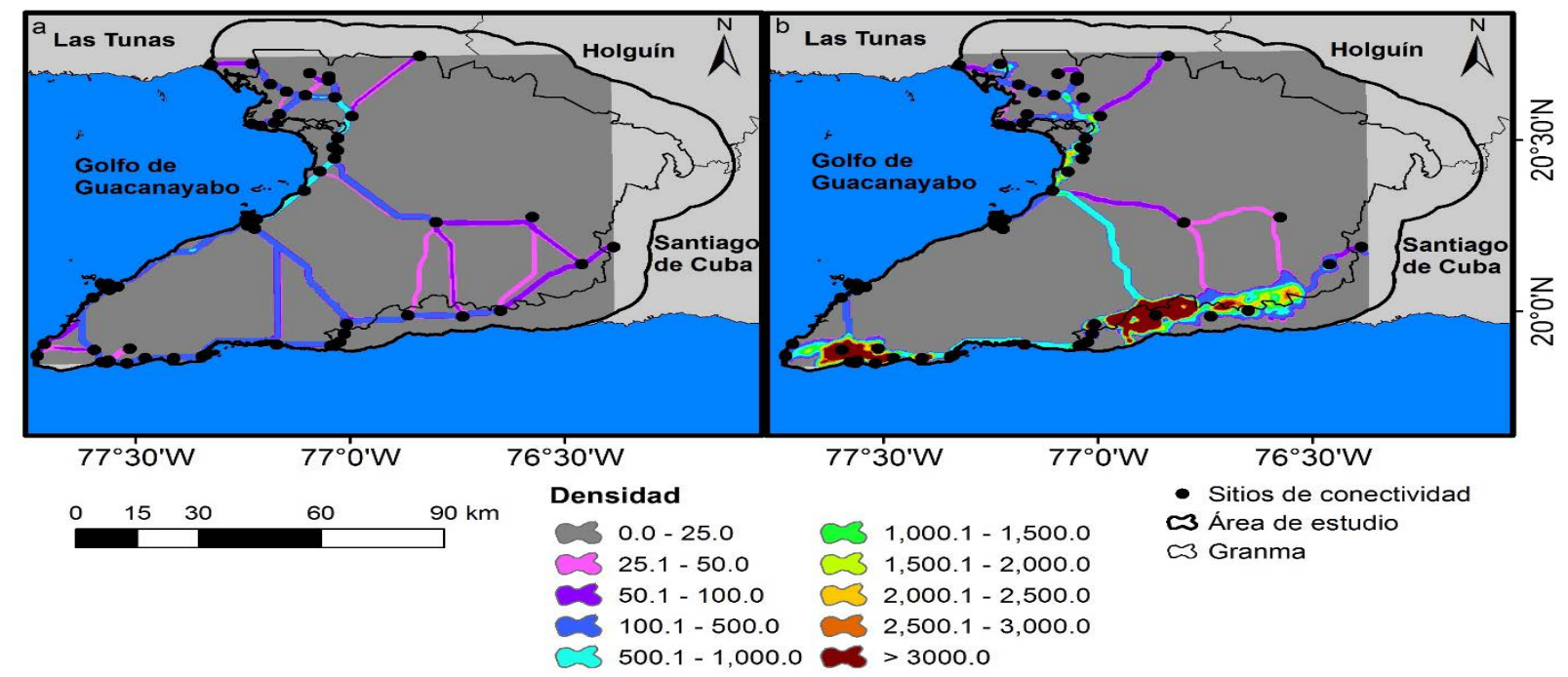

Figura 7. Densidad de rutas de conectividad. a) con el empleo del ráster de costos con rangos, b) con el empleo del ráster de costos sin rangos.

Figure 7. Density of connectivity routes. a) using the cost raster with ranges, b) using the cost raster without ranges.

\begin{tabular}{|c|c|c|}
\hline 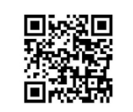 & (c) $\underset{\mathrm{BY}}{(1)}$ (9) $_{\mathrm{SA}}$ & 89 \\
\hline
\end{tabular}




\section{Revista de CIENCIAS AMBIENTALES Tropical Journal of Environmental Sciences}

Revista de Ciencias Ambientales (Trop J Environ Sci)

e-ISSN: 2215-3896 (Enero-Junio, 2022). Vol 56(1): 75-95 DOI: https://doi.org/10.15359/rca.56-1.4 Open Access: www.revistas.una.ac.cr/ambientales e-mail: revista.ambientales@una.ac.cr Rodríguez Cueto Y., Ramón Puebla A.

\subsubsection{Condiciones altitudinales y longitudinales de las rutas identificadas}

Los análisis de las condiciones altitudinales y longitudinales de las rutas identificadas muestran que un total de 518 rutas sufrieron cambios en su condición, al cambiar la forma de obtener el ráster de costos. Dichas 518 rutas (16.8 \% del total identificadas) cambiaron siempre a una condición altitudinal, por lo que el número de rutas altitudinales aumentó en 518, mientras que el número de rutas latitudinales se redujo en ese mismo número. Empleando el método de cálculo del ráster de costos con rangos, la relación entre rutas altitudinales y longitudinales es más homogénea (59\% de rutas altitudinales y $41 \%$ de rutas longitudinales), mientras que con el empleo del método de cálculo de ráster de costos sin rangos esa relación se hace más desequilibrada, a favor de las rutas altitudinales (75.8 \% de rutas altitudinales y $24.2 \%$ de rutas longitudinales).

De acuerdo con lo mostrado en la Figura 8, el $27 \%$ (15) de los sitios de conexión no modificaron su condición altitudinal/longitudinal. El otro $73 \%$ de los sitios incrementan la cantidad de rutas con condición altitudinal. También tres sitios mostraron el mayor número de cambio en la condición, al presentar 34 rutas ( $62 \%$ de las 55 rutas posibles a crearse para cada sitio) que cambiaron a una condición altitudinal, principalmente asociado a la desaparición de las rutas que se generaban en el extremo suroccidental del área de estudio.

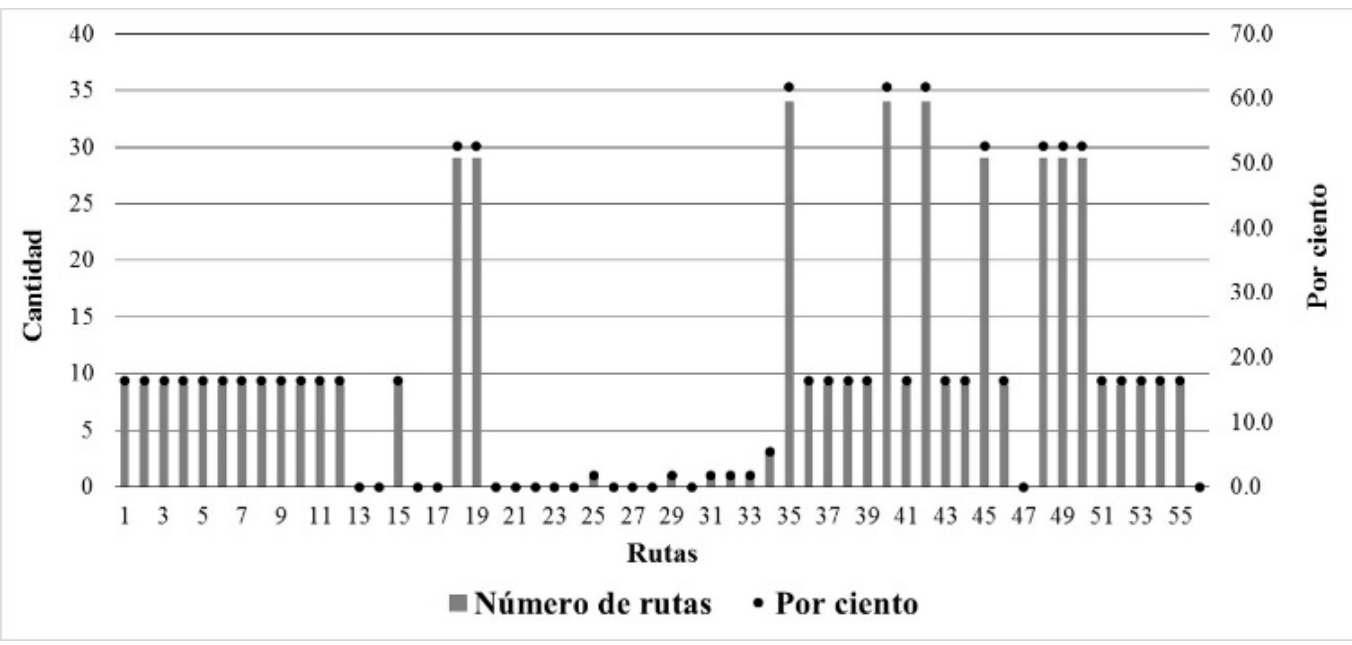

Figura 8. Cambio en la condición altitudinal/longitudinal de las rutas.

Figure 8. Changes in the altitudinal/longitudinal condition of the routes.

Estos cambios poseen implicaciones ecológicas y de conectividad en el área de estudio. Al aumentar el número de rutas altitudinales con el empleo del ráster de costos sin rangos, se incrementa la posibilidad de que las especies puedan moverse de un piso altitudinal a otro, ya sea para aumentar o disminuir la altura respecto al nivel medio del mar a la que se encuentran, siempre y cuando sus condiciones de vida se lo permitan.

\begin{tabular}{|c|c|c|c|c|c|}
\hline 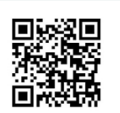 & (c) (1) (9) & $\underset{\text { AMBIENTAES }}{\Leftrightarrow}$ & $\frac{\mid \sigma o \%}{\text { euna }}$ & 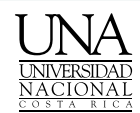 & 90 \\
\hline
\end{tabular}




\section{Revista de CIENCIAS AMBIENTALES Tropical Journal of Environmental Sciences}

Revista de Ciencias Ambientales (Trop J Environ Sci)
e-ISSN: 2215-3896
(Enero-Junio, 2022).Vol 56(1): 75-95
DOI: https://doi.org/10.15359/rca.56-1.4
Open Access: www.revistas.una.ac.cr/ambientales
e-mail: revista.ambientales@una.ac.cr
Rodríguez Cueto Y., Ramón Puebla A.

Aun cuando se producen cambios interesantes en el comportamiento altitudinal/longitudinal de ciertas rutas con el cambio de método de obtención del ráster de costos, hay que señalar que la ubicación de los nodos juega un papel preponderante en dicha condición. Prueba de ello resulta la coincidencia con los resultados de Ramón Puebla et al. (2020) para las rutas de conectividad que, por el sur del área de estudio, unen las zonas ubicadas en las montañas de la Sierra Maestra y la zona suroccidental del área de estudio. La posición de dichos nodos, unos en zonas montañosas y otros en zonas bajas y costeras, genera rutas altitudinales independientemente del método de obtención del ráster de costos al desplazamiento, como muestran nuestros resultados y los de Ramón Puebla et al. (2020).

\section{Conclusiones}

El empleo de ambos métodos de cálculo del ráster de costos, con la presencia de rangos y sin la presencia de estos; muestra diferencias en la identificación de rutas de conectividad. Dichas diferencias se asocian a variaciones en la distribución espacial del costo al desplazamiento, modificaciones en la red de rutas de conectividad, cambios en los sitios de conexión entre regiones en el área de estudio, diferencias en la distribución y los valores de la densidad de rutas, y modificaciones en las condiciones altitudinales y longitudinales de las rutas de conectividad.

Se nota una disparidad en la identificación de nodos de conexión al interior de las ANP, con áreas que presentan gran cantidad de nodos y otras que presentan solamente uno. Ello influye directamente en la posibilidad de conexión de cada ANP con el resto de estas y es una muestra de la fragmentación que existe en las formaciones boscosas al interior de algunas ANP.

El empleo de rangos en la generación del ráster de costos homogeniza el territorio, generando mayor dispersión en la red de rutas de conectividad y disgregando las zonas de conectividad entre diferentes regiones del área de estudio. Ello influye en la densidad de las rutas de conectividad, en la importancia de determinadas ANP para la conectividad, en el trazado de las rutas al interior de los parches de vegetación viables y en la condición altitudinal/longitudinal de las rutas.

Finalmente, se recomienda analizar el efecto del peso y los valores de cada variable empleada sobre el cálculo del ráster de costo, tanto con el uso de rangos como sin el uso de estos, para poder entender la importancia de cada una de estas variables en el costo al desplazamiento en el área de estudio.

\section{5. Ética y conflicto de intereses}

Las personas autoras declaran que han cumplido totalmente con todos los requisitos éticos y legales pertinentes, tanto durante el estudio como en la producción del manuscrito; que no hay conflictos de intereses de ningún tipo; que todas las fuentes financieras se mencionan completa y claramente en la sección de agradecimientos; y que están totalmente de acuerdo con la versión final editada del artículo.

\begin{tabular}{|c|c|c|}
\hline 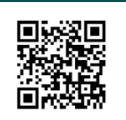 & (c) (i) () () & 91 \\
\hline
\end{tabular}




\section{Revista de CIENCIAS AMBIENTALES Tropical Journal of Environmental Sciences}
Revista de Ciencias Ambientales (Trop J Environ Sci)
e-ISSN: 2215-3896 (Enero-Junio, 2022) . Vol 56(1): 75-95 DOI: https://doi.org/10.15359/rca.56-1.4 Open Access: www.revistas.una.ac.cr/ambientales e-mail: revista.ambientales@una.ac.cr Rodríguez Cueto Y., Ramón Puebla A.

\section{Agradecimientos}

A la Revista y las personas revisoras anónimas por sus oportunos comentarios a la versión final del escrito.

\section{Referencias}

Arias, E., Chacón, O., Herrera, B., Induni, G., Heiner, A., Coto, M., \& Barborak, J. (2008) Las redes de conectividad como base para la planificación de la conservación de la biodiversidad: Propuesta para Costa Rica. Recursos Naturales y Ambiente, 54, 37-43. https://repositorio.bibliotecaorton.catie.ac.cr/handle/11554/6488

Bennett, A. (2004). Enlazando el paisaje: el papel de los corredores y la conectividad en la conservación de la vida silvestre. UICN.

Bennett, G., \& Molungoy, K. J. (2006). Review of experience with ecological networks, corridors and buffer zones (Technical Series No. 23). Secretariat of the Convention on Biological Diversity. https://www.cipra.org/en/publications/3181

Borroto-Páez, R., \& Mancina, C. A. (2011). Mamíferos en Cuba. VASA.

Burkart, S., Gugerli, F., Senn, J., Kuehn, R., \& Bolliger, J. (2016). Evaluating the Functionality of Expert-Assessed Wildlife Corridors with Genetic Data from Roe Deer. Basic and Applied Ecology, 17(1), 52-60. https://doi.org/10.1016/j.baae.2015.09.001

Capote, R., \& Berazaín, R. (1984). Clasificación de las formaciones vegetales de Cuba. Revista del Jardín Botánico Nacional, 2 (mayo), 75-27. https://www.jstor.org/stable/42596743

Convention on Biological Diversity (2016). Indicators for the Strategic Plan for Biodiversity 2011-2020 and the Aichi Biodiversity Targets. https://www.cbd.int/doc/decisions/cop-13/ cop-13-dec-28-en.pdf

Céspedes, M. V., Delgado, D., Velásquez, S., Herrera Fernández, B., Finegan, B., \& Campos Arce, J. (2008). Diseño de una red ecológica de conservación entre la Reserva de Biosfera La Amistad y las áreas naturales protegidas del Área de Conservación Osa, Costa Rica. Recursos Naturales y Ambiente, 54, 44-50. https://repositorio.bibliotecaorton.catie.ac.cr/bitstream/ handle/11554/6057/Diseno_de_una_red.pdf?sequence=1\&isAllowed=y

Chacón Salas, I. (2016) Rutas de conectividad entre las áreas naturales protegidas del Área de Conservación Arenal-Tempisque bajo diferentes escenarios de cambio climático. [Tesis de Maestría, Universidad Nacional]. Repositorio CONICIT. http://repositorio.conicit.go.cr:8080/xmlui/ handle/123456789/244

\begin{tabular}{|c|c|c|}
\hline 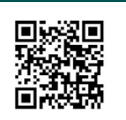 & (c) (i) (5) () & 92 \\
\hline
\end{tabular}




\section{Revista de CIENCIAS AMBIENTALES Tropical Journal of Environmental Sciences}
Revista de Ciencias Ambientales (Trop J Environ Sci)
e-ISSN: 2215-3896 (Enero-Junio, 2022). Vol 56(1): 75-95 DOI: https://doi.org/10.15359/rca.56-1.4 Open Access: www.revistas.una.ac.cr/ambientales e-mail: revista.ambientales@una.ac.cr Rodríguez Cueto Y., Ramón Puebla A.

Colorado Zuluaga, G. J., Vásquez Muñoz, J. L., \& Mazo Zuluaga, I. N. (2017). Modelo de conectividad ecológica de fragmentos de bosque andino en Santa Elena (Medellín, Colombia). Acta Biológica Colombiana, 22(3), 379-393. http://dx.doi.org/10.15446/abc.v22n3.63013

Corredor Jiménez, S. H. (2019). Revisión y comparación del índice de fragmentación de coberturas naturales para cuatro cuencas hidrográficas en estudio. [Proyecto de grado en modalidad investigación-innovación para optar al título de ingeniero, Universidad Distrital Francisco José de Caldas]. Repositorio Institucional Universidad Distrital Francisco José de Caldas. https://repository.udistrital.edu.co/handle/11349/22764

Del Risco, E. (1995). Los bosques de Cuba: su historia y características. Editorial Científico -Técnica.

Environmental Systems Research Institute [ESRI] (2015). ArcGIS Desktop and Spatial Analyst Extension: Release 10.4 [Sofware]. Redlands.

Estrada, R., Martín Morales, G., Martínez Fernández, P., Rodríguez Hernández, S. V., Capote López, R.P., Reyes Alonso, I., Galano Duverger, S., Cabrera Gutiérrez, C., Martínez Bayón, C., Mateo Rego, L., Guerra Sosa, Y., Batte Hernández, A., \& Coya de la Fuente, L. (2011). Mapa (BD-SIG) de vegetación natural y seminatural de Cuba v. 1 sobre Landsat etm 7 slc-off gap filled, circa 2011. [Memorias del IV Congreso de Manejo de Ecosistemas y Biodiversidad]. http:// repositorio.geotech.cu/xmlui/handle/1234/597

Fahrig, L. (2003). Effects of habitat fragmentation on biodiversity. Annual Review of Ecology Evolution and Systematics, 34(1), 487-515. https://doi.org/10.1146/annurev. ecolsys.34.011802.132419

Galpern, P. (2013). Modelling landscape connectivity for highly-mobile terrestrial animals: a continuous and scalable approach. [Tesis de Doctorado, University of Manitoba]. Repositorio Dspace. http://hdl.handle.net/1993/9224

Galpern, P., \& Manseau, M. (2013). Modelling the influence of landscape connectivity on animal distribution: a functional grain approach. Ecography 36, 1001-1016. https://doi. org/10.1111/j.1600-0587.2012.00081.x

Globelandcover 30 (2021). Centro Nacional de Áreas Protegidas. [Base de datos, Imagen]. http:// www.globallandcover.com/home_en.html?type=data

González Alonso, H. (2012). Libro Rojo de los Vertebrados de Cuba. Cuba: Editorial Academia.

González, H. (2002). Aves de Cuba. Finlandia: VAASA.

Hanski, I. (1994). Patch-Occupancy Dynamics in Fragmented Landscapes. Trends in Ecology \& Evolution, 9(4), 131-135. https://doi.org/10.1016/0169-5347(94)90177-5

\begin{tabular}{|c|c|c|c|c|}
\hline 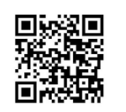 & (c) (1) () (2) & $\underset{\text { AMBENENALIES }}{8}$ & $\frac{10 \%}{\frac{\sigma}{2}}$ & 93 \\
\hline
\end{tabular}




\section{Revista de CIENCIAS AMBIENTALES Tropical Journal of Environmental Sciences}
Revista de Ciencias Ambientales (Trop J Environ Sci)
e-ISSN: 2215-3896
(Enero-Junio, 2022). Vol 56(1): 75-95 DOI: https://doi.org/10.15359/rca.56-1.4 Open Access: www.revistas.una.ac.cr/ambientales e-mail: revista.ambientales@una.ac.cr Rodríguez Cueto Y., Ramón Puebla A.

Herrera Calvo, P. M., \& Díaz Varela, E. R. (2013). Ecología del Paisaje, conectividad ecológica y territorio. Una aproximación al estado de la cuestión desde una perspectiva técnica y científica. Dossier Ciudades, 1, 43-70. https://dialnet.unirioja.es/servlet/articulo? codigo=6826966

Hilty, J. A., Brooks, C., Heaton, E., \& Merenlender, A. M. (2006). Forecasting the effect of landuse change on native and non-native mammalian predator distribution. Biodiversity and Conservation, 15, (9), 2853-2871. https://doi.org/10.1007/s10531-005-1534-5

Iturralde-Vinent, M. (2010). Geología de Cuba para todos. Editorial Científico Técnica

Johnstone, C. P., Lill, A. \& Reina, R. D. (2014). Habitat Loss, Fragmentation and Degradation Effects on Small Mammals: Analysis with Conditional Inference. Tree Statistical Modelling. Biological Conservation, 176, 80-98. https://doi.org/10.1016/j.biocon.2014.04.025

Jun, C., Ban, Y., \& Li, S. (2014). Open access to Earth land-cover map. Nature, 514, 434. https:// doi.org/10.1038/514434c

Kattan, G. (2002). Fragmentación: patrones y mecanismos de extinción de especies. En M. Guariguata, \& G. Kattan (Eds). Ecología y Fragmentación de Bosques Tropicales. Ediciones LUR.

Mancina, C., \& Cruz, D. (Eds.). (2017). Diversidad biológica de Cuba. Métodos de inventario, monitoreos y colecciones biológicas. Editorial AMA.

Mateo, J. (2017). Regiones y paisajes geográficos de Cuba. Primera Parte: Macro región oriental. Ministerio de Educación Superior.

Mindreau, M. (2013). Aspectos Conceptuales sobre el Diseño Biofísico de las Redes de Conectividad. SERNANP.

Newmark, W. D. (2001). Tanzania forest edge microclimatic gradients: dynamic patterns. Biotropica, 33, 2-11. https://doi.org/10.1646/0006-3606(2001)033[0002:tfemgd]2.0.co;2

Oficina Nacional de Estadísticas e Información. (2019). Anuario Estadístico de Cuba. Cuba.

Peled, E., Shanas, U., Granjon, L., \& Ben-Shlomo, R. (2016). Connectivity in Fragmented Landscape: Generalist and Specialist Gerbils Show Unexpected Gene Flow Patterns. Journal of Arid Environments, 125, 88-97. 10.1016/j.jaridenv.2015.10.006

Ramón Puebla, A., Rodríguez Cueto, Y., \& Álvarez-Amargos, P. (2020). Propuesta de rutas de conectividad para conservación de la biodiversidad en Sierra Maestra. Cuba. Revista de Ciencias Ambientales, 54(2), 51-67. https://doi.org/10.15359/rca.54-2.3

Sawyer, S. C., Epps, C. W., \& Brashares, J. S. (2011). Placing linkages among fragmented habitats: do least-cost models reflect how animals use landscapes? Journal of Applied Ecology, 48, 668678. https://doi.org/10.1111/j.1365-2664.2011.01970.x

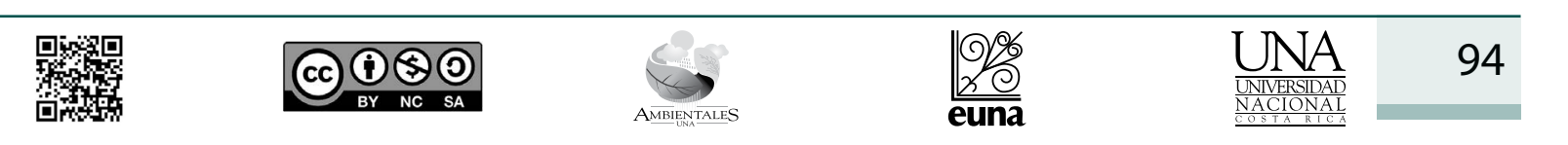




\section{Revista de CIENCIAS AMBIENTALES

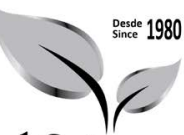 Tropical Journal of Environmental Sciences}
Revista de Ciencias Ambientales (Trop J Environ Sci) e-ISSN: 2215-3896 (Enero-Junio, 2022) . Vol 56(1): 75-95 DOI: https://doi.org/10.15359/rca.56-1.4 Open Access: www.revistas.una.ac.cr/ambientales e-mail: revista.ambientales@una.ac.cr Rodríguez Cueto Y., Ramón Puebla A.

Urban, D., \& Keitt, T. (2001). Landscape connectivity: A graph-theoretic perspective. Ecology, 82, 1205-1218. https://doi.org/10.1890/0012-9658(2001)082[1205:LCAGTP]2.0.CO;2

Zeller, K. A., K. McGarigal, \& Whiteley, A. R. (2012). Estimating landscape resistance to movement: a review. Landscape Ecology, 27, 777-797. 10.1007/s10980-012-9737-0 